\title{
Caudal, a key developmental regulator, is a DPE-specific transcriptional factor
}

\author{
Tamar Juven-Gershon, Jer-Yuan Hsu, and James T. Kadonaga ${ }^{1}$ \\ Section of Molecular Biology, University of California at San Diego, La Jolla, California 92093, USA
}

\begin{abstract}
The regulation of gene transcription is critical for the proper development and growth of an organism. The transcription of protein-coding genes initiates at the RNA polymerase II core promoter, which is a diverse module that can be controlled by many different elements such as the TATA box and downstream core promoter element (DPE). To understand the basis for core promoter diversity, we explored potential biological functions of the DPE. We found that nearly all of the Drosophila homeotic (Hox) gene promoters, which lack TATA-box elements, contain functionally important DPE motifs that are conserved from Drosophila melanogaster to Drosophila virilis. We then discovered that Caudal, a sequence-specific transcription factor and key regulator of the Hox gene network, activates transcription with a distinct preference for the DPE relative to the TATA box. The specificity of Caudal activation for the DPE is particularly striking when a BRE $^{\mathrm{u}}$ core promoter motif is associated with the TATA box. These findings show that Caudal is a DPE-specific activator and exemplify how core promoter diversity can be used to establish complex regulatory networks.
\end{abstract}

[Keywords: Core promoter; RNA polymerase II transcription; DPE; Hox; Caudal; BRE ${ }^{\mathrm{u}}$ ]

Supplemental material is available at http://www.genesdev.org.

Received May 21, 2008; revised version accepted August 25, 2008.

Sequence-specific DNA-binding transcription factors play a key role in the control of many biological phenomena, such as in the development of the body plan of Drosophila melanogaster (Nüsslein-Volhard and Wieschaus 1980; Akam 1987; Gehring 1987; Pearson et al. 2005; Lappin et al. 2006; Lemons and McGinnis 2006; Ochoa-Espinosa and Small 2006). A simple view of this process is as follows: Maternal genes such as bicoid and caudal establish the anteroposterior axis of the embryo; gap genes such as hunchback, giant, Krüppel, and knirps subdivide the embryo into broad regions; pair-rule genes such as fushi tarazu specify the number of segments; segment polarity genes such as engrailed determine the anteroposterior polarity of each segment; and homeotic (Hox) genes such as Antennapedia specify the identity of the segments. Remarkably, the vast majority of these genes encode sequence-specific DNA-binding transcription factors that bind to promoter and enhancer elements and regulate transcription by RNA polymerase II. It remains important to investigate, however, whether these developmental regulators act with specificity not only at promoters and enhancers, but also at the site of transcription initiation, the core promoter.

The RNA polymerase II core promoter is a diverse transcriptional module that directs the initiation of transcription (for review, see Smale 2001; Smale and Kadonaga 2003; Juven-Gershon et al. 2008). Core promoters

${ }^{1}$ Corresponding author.

E-MAIL jkadonaga@ucsd.edu; FAX (858) 534-0555.

Article is online at http://www.genesdev.org/cgi/doi/10.1101/gad.1698108. may contain one or more core promoter motifs, which include DNA sequence elements such as the TATA box, TFIIB recognition elements $\left(\mathrm{BRE}^{\mathrm{u}}\right.$ and $\left.\mathrm{BRE}^{\mathrm{d}}\right)$, initiator (Inr), motif 10 element (MTE), and the downstream core promoter element (DPE). There are no universal core promoter motifs.

The existence of many different types of core promoters suggests that their functions extend beyond the specification of transcription initiation. Consistent with this notion, it has been found that core promoter motifs can contribute to enhancer-promoter specificity (for review, see Smale 2001; Butler and Kadonaga 2002). For instance, the Drosophila AE1 and IAB5 enhancers exhibit a preference for activation of the TATA-containing even-skipped core promoter relative to the TATA-less and DPE-containing white core promoter (Ohtsuki et al. 1998). In addition, enhancer trapping studies in Drosophila led to the discovery of DPE-specific transcriptional enhancers (Butler and Kadonaga 2001). Thus, core promoter diversity provides an additional dimension to the constellation of mechanisms by which genes are regulated. At the level of basal transcription, it has been found that factors such as NC2, Mot1, and TBP affect DPE-dependent versus TATA-dependent transcription (for example, see Hsu et al. 2008). However, for regulated transcription, the enhancer-binding factors that mediate core promoter specificity have not yet been identified.

To identify biological functions of core promoter motifs, we explored the potential role of the DPE in transcriptional regulatory networks. The DPE was originally 
discovered as a TFIID recognition site that is located downstream from the Inr element (Burke and Kadonaga 1996). The DPE is conserved from Drosophila to humans (Burke and Kadonaga 1997). TFIID binds cooperatively to the Inr and DPE, which is located precisely at +28 to +32 relative to $\mathrm{A}+1$ in the Inr consensus sequence. The correct spacing between the Inr and DPE is critical for optimal transcriptional activity (Kutach and Kadonaga 2000). Current evidence suggests that the DPE is present in $\sim 2.1 \%-22 \%$ of Drosophila core promoters (Ohler et al. 2002; FitzGerald et al. 2006; Gershenzon et al. 2006).

In this study, we identify a critical role of the DPE motif in the transcription of genes that are involved in the early embryonic development of Drosophila. We further discovered that Caudal, a master regulator of the Hox genes, is a DPE-specific enhancer-binding factor. These findings reveal the use of the DPE as a component of the regulatory circuitry of an important biological process and uncover a new mechanism with which sophisticated patterns of gene expression are established to achieve organismal complexity (for example, see Levine and Tjian 2003).

\section{Results}

The core promoters of nearly all Drosophila Hox genes contain conserved DPE motifs

To uncover a specific biological role of the DPE, we looked for patterns or related themes in Drosophila genes whose core promoters have been shown to possess or are believed to contain DPE motifs. This analysis led us to the genes that are involved in the early development of the embryo. In the course of our previous studies, Antennapedia (Antp; downstream P2 promoter), engrailed (en), and caudal (cad; zygotic promoter) were among the genes whose promoters had been found to contain functionally important DPE motifs (Burke and Kadonaga 1996; Kutach and Kadonaga 2000), but a correlation between the DPE and developmental genes was not yet apparent. However, with the current Drosophila genome data (http://www.fruitfly.org; http://flybase.net; http://genome.ucsc.edu/cgi-bin/hgGateway), we found that the core promoters of many developmentally impor- tant genes, particularly the Hox genes, appear to contain DPE motifs. The Hox genes encode sequence-specific transcription factors that bind to DNA via their conserved homeodomain and are key regulators of the development of the embryonic body plan. The promoters of the Hox genes are TATA-less; hence, the DPE motifs could support Hox gene transcription in the absence of the TATA box.

To assess the potential biological significance of the DPE motifs in the Hox genes, we examined the conservation of the DPE sequences in eight different Drosophila species. The Inr and DPE consensus sequences as well as the Inr-to-DPE spacing are conserved across species in the labial (lab), proboscipedia $(p b)$, Deformed $(D f d)$, Sex combs reduced (Scr), Antennapedia P1 (Antp P1), Antennapedia P2 (Antp P2), and Abdominal-B (Abd$B$ ) core promoters (Supplemental Fig. 1; data not shown). The conservation of these core promoter sequences between $D$. melanogaster and Drosophila virilis, which are separated by an evolutionary period of 40-60 million years, is shown in Figure 1. Only two Drosophila Hox genes, Abdominal-A (Abd-A) and Ultrabithorax (Ubx), do not contain DPE consensus motifs. However, $A b d-A$ and $U b x$ have arisen more recently than the other Hox genes (Grenier et al. 1997; Brooke et al. 1998); hence, all of the more ancient Hox genes contain DPE consensus sequences.

We then tested whether the DPE consensus sequences in the Hox genes are functionally important for transcription. To this end, we constructed and analyzed wild-type and mutant DPE (mDPE) versions of the Hox gene core promoters with putative DPE sequences. These experiments showed that all of the D. melanogaster Hox genes, except for $A b d-A$ and $U b x$, contain functionally important DPE motifs (Fig. 2A; Supplemental Fig. 2).

\section{Caudal, a key regulator of the Hox gene network, activates DPE-dependent promoters and some but not all TATA-dependent promoters}

The prevalence of the DPE in the core promoters of the Hox genes suggests that some of the transcriptional regulatory factors that activate Hox gene expression are DPE-

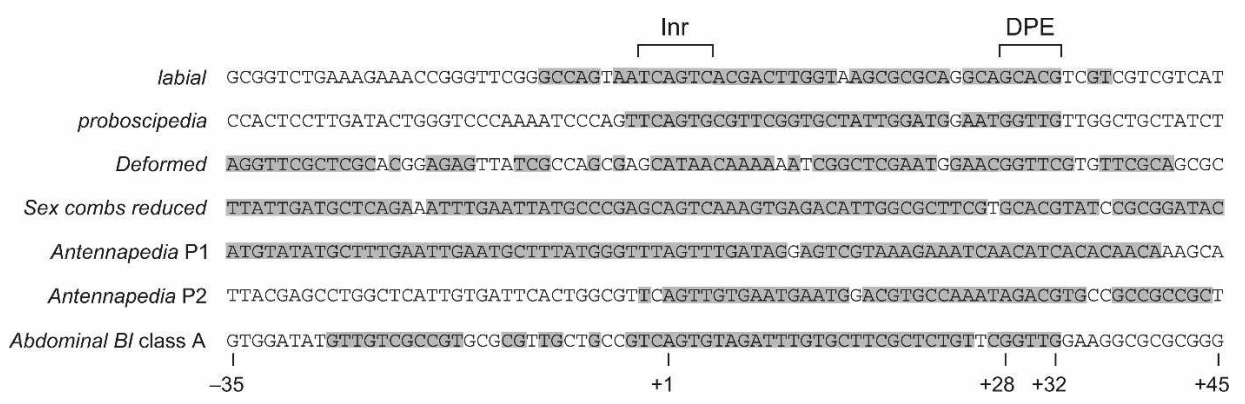

Figure 1. The core promoters of Drosophila Hox genes contain a conserved DPE motif. The core promoter sequences of the indicated D. melanogaster Hox genes are shown. We initially analyzed the sequence conservation of the promoters in eight different Drosophila species by using VISTA tools and CLUSTALW (Supplemental Fig. 1; data not shown). Based on this sequence alignment, positions that are identical between $D$. melanogaster and $D$. virilis (in terms of sequence and spacing relative to the $\mathrm{A}+1 \mathrm{in}$ the Inr) are shaded. $D$. melanogaster and $D$. virilis are estimated to be separated by an evolutionary period of $\sim 40-60$ million years. 
A

\section{lab $\quad p b$ Dfd Scr Antp Ubx abd-A Abd-B}
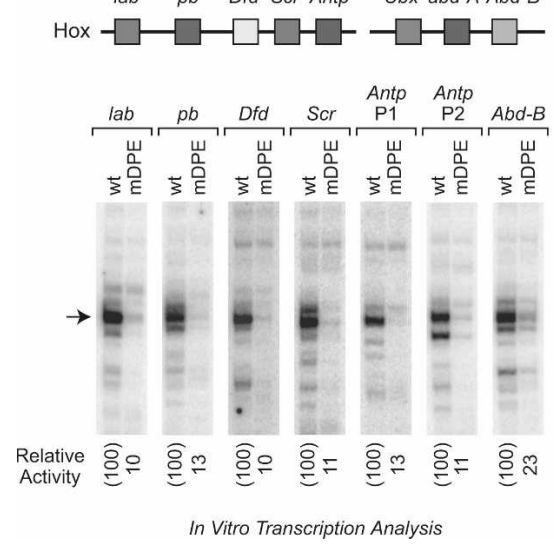

B

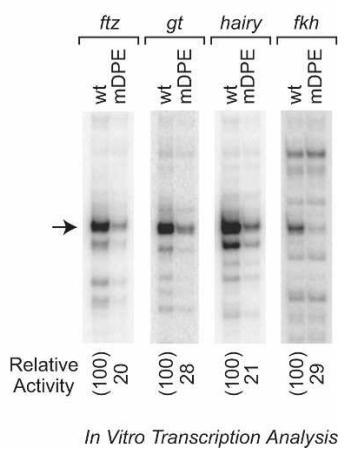

Figure 2. The DPE is present in many genes that regulate the development of the Drosophila body plan. $(A)$ Most of the Hox genes contain DPE-dependent core promoters. A schematic diagram of the Hox gene cluster is shown at the top. (lab) labial; $(p b)$ proboscipedia; $(D f d)$ Deformed; (Scr) Sex combs reduced; (Antp P1) Antennapedia upstream promoter; (Antp P2) Antennapedia downstream promoter; $(U b x)$ Ultrabithorax; $(a b d-A) a b$ dominal- $A_{;}(A b d-B)$ Abdominal-B. The wild-type (wt) and mutant DPE (mDPE) versions of the indicated core promoters (from -10 to +40 relative to the $A+1$ start site) were subjected to in vitro transcription analysis with a Drosophila embryo nuclear extract. The resulting transcripts were detected by primer extension-reverse transcription analysis. $(B)$ The core promoters of Caudal target genes contain functional DPE motifs. (ftz) fushi tarazu; (gt) giant; (h) hairy; (fkh) forkhead. In vitro transcription analysis was performed as in $A$. specific activators. To investigate this hypothesis, we examined the protein encoded by the caudal (cad) gene. cad is a paralog of the Hox genes and is thus termed a ParaHox gene (Brooke et al. 1998). cad was first identified in $D$. melanogaster and is expressed both maternally and zygotically (Mlodzik et al. 1985; Mlodzik and Gehring 1987). It is required for the specification of the posterior embryo and patterning of the anteroposterior axis (Levine et al. 1985; Mlodzik et al. 1985; Hoey et al. 1986; Macdonald and Struhl 1986; Mlodzik and Gehring 1987). Caudal protein contains a homeodomain and is a sequence-specific DNA-binding activator. In vertebrates, the Caudal proteins ( $\mathrm{Cdx} 1, \mathrm{Cdx} 2$, and $\mathrm{Cdx} 4)$ are master regulators of Hox gene expression (for example, see Charité et al. 1998; Davidson et al. 2003; Lohnes 2003; Davidson and Zon 2006).

In D. melanogaster, Caudal has been observed to regulate the developmentally important genes fushi tarazu (ftz) (Dearolf et al. 1989), giant (gt) (Rivera-Pomar et al. 1995; Schulz and Tautz 1995; Rivera-Pomar and Jäckle 1996), hairy (h) (Rivera-Pomar et al. 1995; Rivera-Pomar and Jäckle 1996), and forkhead (fkh) (Wu and Lengyel 1998). Notably, the core promoters of these Caudal target genes appear to contain DPE motifs. We constructed and analyzed the wild-type and mutant DPE versions of the $f t z, g t, h$, and $f k h$ core promoters and found that they all contain functional DPE motifs (Fig. 2B). These findings suggest that Caudal might be a DPE-specific activator.

To test whether Caudal selectively activates transcription from DPE-dependent core promoters, we developed an assay system (Fig. 3A) that involves cotransfection of a Caudal expression plasmid along with a DPE- or TATA-dependent reporter gene in D. melanogaster Schneider (S2) cells, which contain negligible levels of cad transcripts (data not shown). The DPE and TATA reporter constructs contain well-characterized TATA and DPE motifs along with six Caudal-binding sites from the $f t z$ enhancer (Dearolf et al. 1989) and are identical except for the sequences at the DPE and TATA regions. These experiments revealed that Caudal strongly activates transcription with the Antp P2 DPE, but only weakly activates transcription with the adenovirus major late (AdML) TATA box (Fig. 3A).

The differential effect of Caudal on the two reporter genes is not due to differences in core promoter strength (Supplemental Fig. 3). We also observed that strong activation by Caudal is dependent on the presence of Caudal-binding sites in the reporter gene (Supplemental Fig. 4). In addition, the inability of Caudal to activate the TATA-dependent construct is not due to a defect in the ability of the promoter to respond to activators, because reporter constructs with the Antp P2 DPE or the AdML TATA box are both activated by the Alcohol dehydrogenase (Adh) distal enhancer (Fig. 3B).

To investigate the generality of Caudal activation of DPE-dependent core promoters, we constructed and analyzed a different set of DPE- and TATA-dependent reporter genes with the $E 74 B \mathrm{DPE}$ and the Adh proximal promoter TATA box. We found that Caudal activates transcription not only with the $E 74 B$ DPE motif, but also with the Adh TATA box (Fig. 3C). Given the results with the AdML TATA box (Fig. 3A), the activation of the Adh TATA-dependent reporter by Caudal was surprising. We therefore tested different combinations of the Adh and AdML TATA boxes and the Antp P2 and E74B DPE motifs and found that Caudal activates transcription with either DPE motif as well as with the Adh TATA box, but not with the AdML TATA box (Fig. 3; Supplemental Fig. 5). Therefore, Caudal activates transcription from DPEdependent promoters and some but not all TATA-dependent promoters.

The BRE core promoter motif suppresses the ability of Caudal to activate TATA-dependent promoters

To determine the basis for Caudal activation with the Adh TATA but not the AdML TATA, we examined the promoter sequences and observed that the AdML TATA is flanked by $\mathrm{BRE}^{\mathrm{u}}$ and $\mathrm{BRE}^{\mathrm{d}}$ motifs, whereas the $A d h$ TATA region lacks both BRE motifs. BRE sequences flank a subset of TATA-box elements and are sites of interaction with TFIIB (Lagrange et al. 1998; Deng and Roberts 2005). Depending on the context, the BRE motifs 
Juven-Gershon et al.

A

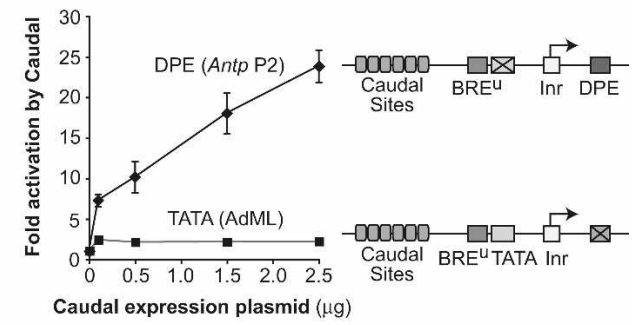

B

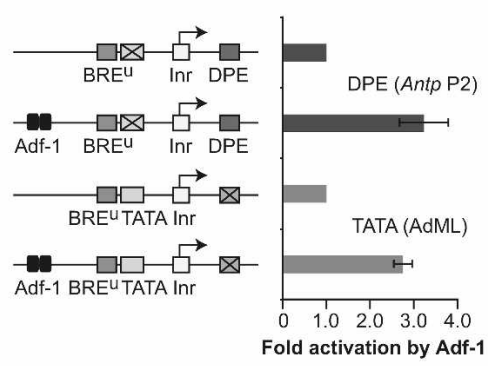

C

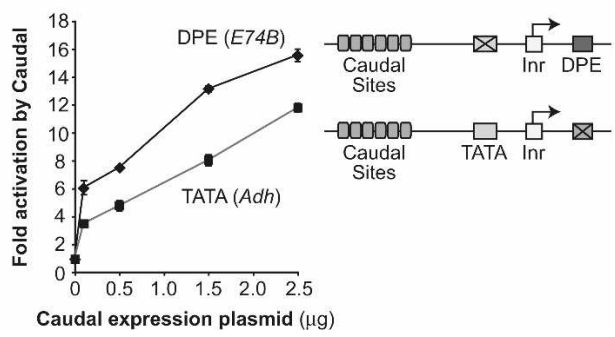

Figure 3. Caudal, a conserved master regulator of the Hox gene network, is a core-promoter-specific activator. $(A)$ Caudal activates transcription with the Antp P2 DPE motif but not with the AdML TATA element. The two reporter constructs are identical except for the TATA and DPE sequences. Drosophila S2 cells were transfected with firefly luciferase reporter constructs as well as a Caudal expression plasmid, where indicated. To normalize for transfection efficiency, cells were cotransfected with a Pol III-Renilla luciferase control plasmid and assayed for dual luciferase activity. Error bars represent the SEM. $(B)$ The Adh distal enhancer activates transcription with both the AdML TATA box and the Antp P2 DPE motif. The four reporter constructs are identical except for the presence or absence of the TATA, DPE, and Adh distal enhancer, as indicated. The Adh distal enhancer contains two binding sites for the Adf- 1 transcription factor, which is present in S2 cells. Transfection assays were performed with the indicated constructs as in $A$. Error bars represent the SEM. $(C)$ Caudal activates transcription with the $E 74 B$ DPE motif and, to a lesser extent, the Adh proximal TATA element. Transfection assays were performed with the indicated constructs as in $A$. Error bars represent the SEM.

can have a positive or negative influence upon transcription (Deng and Roberts 2007).

We tested whether the addition of $\mathrm{BRE}^{\mathrm{u}}$ or $\mathrm{BRE}^{\mathrm{d}}$ sequences to the Adh TATA box affects the ability of Caudal to activate transcription. The addition of a BRE ${ }^{\mathrm{u}}$ motif to the Adh TATA box renders the TATA box unresponsive to activation by Caudal (Fig. 4). In contrast, the presence or absence of the $\mathrm{BRE}^{\mathrm{d}}$ (with or without a BRE ${ }^{\mathrm{u}}$ ) had little effect on Caudal activation (data not shown).
These experiments show that transcriptional activation by Caudal is suppressed by the presence of a BRE ${ }^{\mathrm{u}}$ motif upstream of the TATA box. This novel activity of the $\mathrm{BRE}^{\mathrm{u}}$ core promoter motif may be an additional means of preventing undesired gene activation by Caudal.

Caudal is a DPE-specific activator with the natural ftz enhancer-promoter region

Next, we sought to characterize the ability of Caudal to activate transcription with core promoters from natural Caudal target genes instead of hybrid core promoters. We first analyzed the $g t$ core promoter. $g t$ is a gap gene that is zygotically expressed in the early embryo and is involved in the initial steps in the segmentation of the embryo (Rivera-Pomar et al. 1995; Schulz and Tautz 1995; Rivera-Pomar and Jäckle 1996). The gt core promoter contains TATA and DPE motifs and is activated by Caudal in S2 cells (Supplemental Fig. 6). To determine the relative contribution of the DPE versus TATA motifs to the activation of transcription by Caudal, we compared a DPE-dependent version of the $g t$ promoter (which has a mutant TATA box) with a TATA-dependent version of the promoter (which has a mutant DPE). These experiments show that Caudal preferentially activates transcription through the DPE in the gt core promoter (Fig. 5A). We also found, consistent with the results in Figure 4, that the selectivity of Caudal for activation of DPE- relative to TATA-dependent promoters is further enhanced by the presence of a BRE ${ }^{\mathrm{u}}$ motif upstream of the TATA box (Fig. 5A). In contrast, the BRE motif does not affect the ability of Caudal to activate transcription with the DPE motif (Supplemental Fig. 6). As with the hybrid promoter constructs (Supplemental Fig. 3), the differential ability of Caudal to activate the reporter genes is not due to differences in core promoter strength (Supplemental Fig. 6).

Lastly, we examined the ability of Caudal to activate transcription with the natural enhancer and promoter region of the $f t z$ gene, which is a direct target of Caudal with known binding sites (Dearolf et al. 1989). ftz is a pair-rule gene that is involved in the specification of the number of segments in the embryo. We analyzed the $f t z$ enhancer and promoter region to characterize Caudal ac-

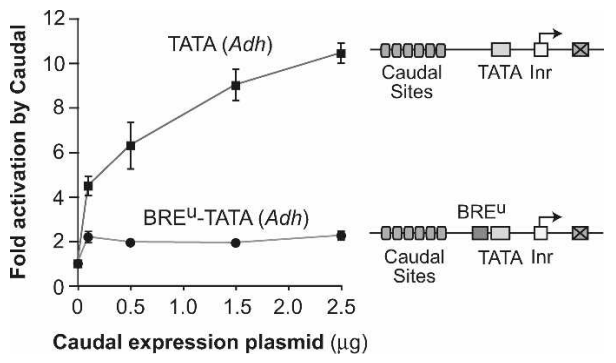

Figure 4. Transcriptional activation by Caudal is suppressed by the presence of a BRE ${ }^{u}$ motif upstream of the TATA box. The two reporter constructs are identical except for the absence or presence of the AdML BRE motif immediately upstream of the TATA box, and were analyzed as in Figure 3. Error bars represent the SEM. 
A
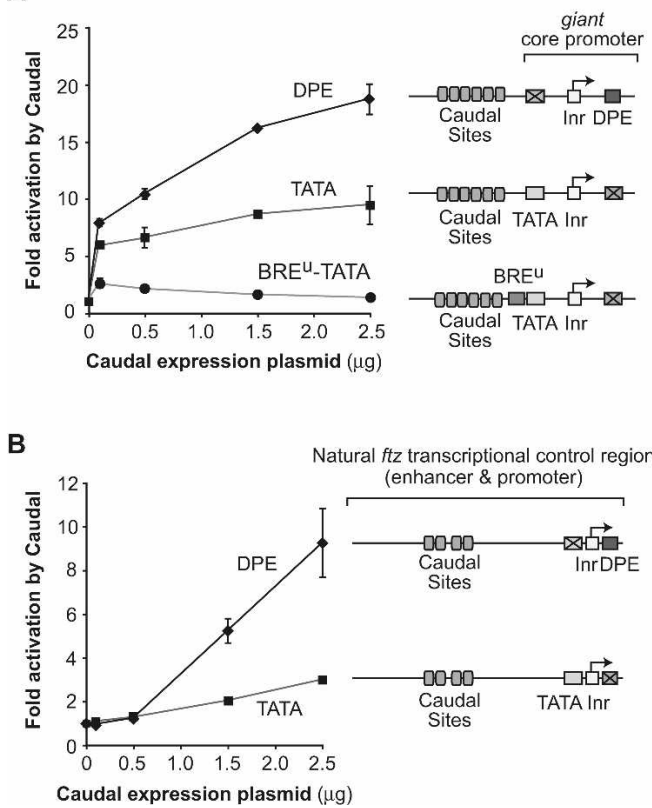

Figure 5. Caudal is a DPE-specific transcriptional activator. (A) Caudal preferentially activates transcription through the DPE in the $g t$ core promoter. The reporter constructs contain six Caudal-binding sites upstream of the $g t$ core promoter, which has both DPE and TATA motifs but lacks a BRE ${ }^{\mathrm{u}}$. The constructs are identical except for the mutation of the TATA and DPE motifs and the addition of the AdML BRE ${ }^{\mathrm{u}}$, where indicated. The transfection experiments were performed as in Figure 3. Error bars represent the SEM. (B) Caudal primarily activates through the DPE in the natural $f t z$ transcriptional control region. The $f t z$ core promoter contains both DPE and TATA motifs. The reporter constructs contain $f t z$ enhancer and promoter sequences from -988 to +40 relative to the +1 start site, and are identical except for mutation of the DPE or TATA, as depicted. The transfection experiments were performed as in Figure 3. Error bars represent the SEM.

tivation in a natural context, as it is possible that Caudal activation with six tandem binding sites upstream of the TATA box (as in Figs. 3, 4, 5A) is more potent than that in a typical Caudal response gene. The $f t z$ core promoter contains both TATA and DPE motifs that are functional in vivo (Supplemental Fig. 7). To determine the relative contributions of the DPE versus the TATA box to Caudal activation, we compared the properties of DPE-dependent (with mutant TATA) versus TATA-dependent (with mutant DPE) versions of the $f t z$ transcriptional control region from -988 to +40 relative to the RNA start site. These studies reveal that Caudal-mediated activation of the $f t z$ gene occurs primarily via the DPE motif in the core promoter (Fig. 5B). Therefore, in the context of a natural transcriptional control region, Caudal is a DPEspecific transcriptional activator.

\section{Caudal activates the TATA-less, DPE-dependent Antp P2 and Scr promoters}

We observed that most of the Drosophila Hox genes contain DPE motifs and that Caudal is a DPE-specific acti- vator. We therefore tested whether Caudal activates transcription from DPE-containing Hox genes. To this end, we isolated Antp P2 and Scr genomic DNA fragments that contain at least $3 \mathrm{~kb}$ of sequence upstream of the A +1 site in the Inr. Both the Antp P2 and Scr core promoters contain DPE and Inr motifs and lack TATA boxes. To determine the effect of the DPE on transcription from these promoters, we made a parallel set of constructs in which the DPE sequences are mutated. Transfection analysis of the wild-type and mutant DPE versions of the Antp P2 and Scr promoters revealed that both promoters are activated by Caudal and are strongly dependent on the DPE motif in cultured cells (Fig. 6). Thus, the DPE is a critical component in Caudal-mediated activation of the natural Antp P2 and Scr promoters.

\section{Discussion}

The DPE is a transcriptional element shared by most Hox genes

In this study, we found that the DPE is used extensively in the network of genes that are involved in the devel-

A

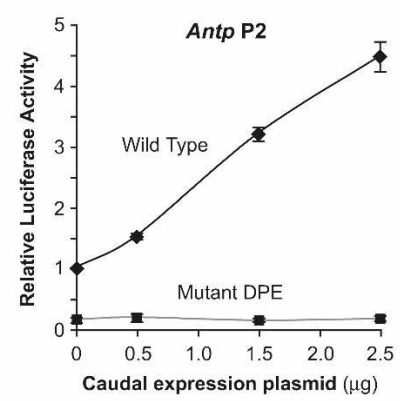

B

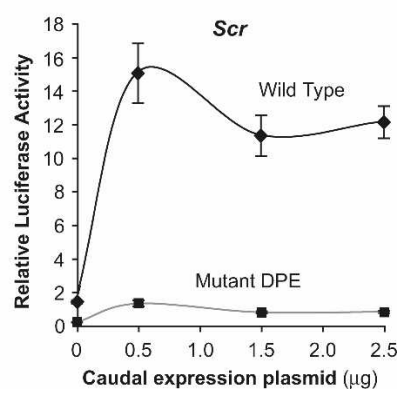

Figure 6. The natural Antp P2 and Scr promoters are activated by Caudal and are dependent on the DPE core promoter motif in S2 cells. (A) Antp P2 promoter. Constructs containing the wildtype and mutant versions of the Antp P2 promoter region (from -3033 to +61 relative to $A+1$ in the Inr) were analyzed as in Figure 3 . The activities are reported relative to the wild-type Antp P2 promoter in the absence of cotransfected Caudal expression plasmid, which was defined to be 1. Error bars represent the SEM. (B) Scr promoter. Constructs containing the wildtype and mutant versions of the $S c r$ promoter region (from -3103 to +110 relative to $\mathrm{A}+1$ in the Inr) were analyzed as in Figure 3. The activities are reported relative to the wild-type Antp P2 promoter in the absence of cotransfected Caudal expression plasmid, which was defined to be 1. Error bars represent the SEM. 
opment of the early Drosophila embryo. Nearly all of the Drosophila Hox gene promoters, which have been known to be TATA-less, contain functionally essential DPE motifs that are conserved from $D$. melanogaster to $D$. virilis. The two Hox genes lacking DPE motifs are also the most recent Hox genes from an evolutionary standpoint. Thus, the DPE is a critical yet previously unrecognized component of the Hox genes.

\section{Caudal is a DPE-specific enhancer-binding factor}

The DPE is not only in the Hox genes, but is also present in $f t z, g t, h, f k h, c a d$ (zygotic promoter), and en, which are involved in early embryonic development. This finding suggests that the DPE is used broadly throughout the network of genes that mediate the development of the embryo. We tested this hypothesis by analyzing the transcriptional properties of Caudal, a ParaHox protein and sequence-specific DNA-binding factor that regulates $f t z$, $g t, h$, and $f k h$. These studies revealed that Caudal is a DPE-specific activator. The preference of Caudal for activating transcription from DPE- versus TATA-dependent core promoters is seen most distinctly either with the natural $f t z$ enhancer-promoter region (Fig. 5B) or with a core promoter containing a $\mathrm{BRE}^{\mathrm{u}}$ motif associated with the TATA box (Figs. 3A, 5A). We also examined the effect of Caudal on transcription of two Hox genes, Antp and $S c r$, and found that Caudal activates the TATA-less, DPE-dependent Antp P2 and Scr promoters (Fig. 6). These findings thus provide a direct link between Caudal, a DPE-specific activator, and the DPE-containing Hox genes.

The discovery that Caudal is a DPE-specific activator provides new insight into the basic mechanisms of transcriptional regulation. Previous enhancer-trapping experiments have shown that there are enhancers that activate DPE-dependent promoters but not TATA-dependent promoters (Butler and Kadonaga 2001); however, neither the cis-acting elements nor the trans-acting factors that are responsible for the DPE-specific activation had been identified. Therefore, these studies demonstrate the existence of a DPE-specific enhancer-binding factor. Moreover, it is likely that other core-promoterspecific enhancer-binding factors, such as TATA-specific activators, will be discovered.

\section{A novel activity of the BRE core promoter motif}

These experiments uncovered a novel activity of the $\mathrm{BRE}^{\mathrm{u}}$ core promoter motif. The $\mathrm{BRE}^{\mathrm{u}}$ is a $5^{\prime}$ extension of the TATA box that is bound by the TFIIB basal/general transcription factor (Lagrange et al. 1998). Depending on the context, the $\mathrm{BRE}^{\mathrm{u}}$ has been found to have either a positive or a negative effect on transcription (Lagrange et al. 1998; Evans et al. 2001). In this study, we found that the $\mathrm{BRE}^{\mathrm{u}}$ motif has little effect on basal/unactivated transcription, but potently suppresses the ability of Caudal to activate transcription via the TATA box. In contrast, the $\mathrm{BRE}^{\mathrm{u}}$ in its normal upstream location has no effect on Caudal-mediated activation via the DPE (Supplemental Fig. 6). These findings indicate that the $\mathrm{BRE}^{\mathrm{u}}$ can contribute to core-promoter-element-mediated transcriptional regulation. Hence, there is a positive linkage between Caudal and the DPE as well as a negative interaction between Caudal and the BRE ${ }^{\mathrm{u}}$-TATA element. The combination of both positive (DPE) and negative (BRE $\left.{ }^{\mathrm{u}}-\mathrm{TATA}\right)$ interactions yields maximal specificity of Caudal function.

The use of core promoter motif specificity in transcriptional circuits

The new findings lead to the model that transcriptional regulation involves the combined action of sequence motifs in both the core promoter and the enhancer. The ability of Caudal to discriminate among DPE, TATA, and $\mathrm{BRE}^{\mathrm{u}}$-TATA motifs regulates the flow of information from the enhancer-bound activator to the core promoter-the site of transcription initiation. In this manner, core promoter elements can be viewed as a component of transcriptional circuits (Fig. 7). In these transcriptional circuits, connections between enhancers and core promoters are established and modulated according to the properties of the activators and the sequence motifs in the core promoter. Thus, the discovery that Caudal is a core-promoter-specific activator reveals a new strategy with which complex transcriptional networks can be established. Hence, in a broader sense, these findings show how diversity in core promoter structure can contribute to organismal diversity.

\section{Materials and methods}

Sequence conservation analysis

Sequence conservation analysis was performed using VISTA tools and CLUSTALW.

\section{In vitro transcription assays}

Double-stranded oligonucleotides comprising sequences from -10 to +40 of the core promoters (relative to $\mathrm{A}+1$ in the Inr) were inserted into the PstI and XbaI sites of pUC119. Mutation of the DPE in the core promoters was identical to that used previously (Lim et al. 2004), where the mutant DPE has CATA at +30 to +33 relative to $\mathrm{A}+1$. In vitro transcription reactions were carried out as described previously (Wampler et al. 1990)

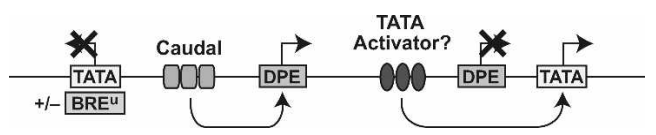

Figure 7. A model for DPE-specific activation of transcription by Caudal. This model depicts a hypothetical segment of a regulatory network that comprises specific connections between activators and core promoter motifs. Caudal is a DPE-specific activator. TATA-specific activators might also exist. The ability of Caudal to function with a TATA box is further reduced by the presence of a BRE ${ }^{u}$ motif, which is located immediately upstream of a subset of TATA elements. 
by using $250 \mathrm{ng}$ of supercoiled DNA templates with Drosophila high salt nuclear extracts (Soeller et al. 1988). The resulting transcripts were subjected to primer extension analysis with M13 reverse sequencing primer (AGCGGATAACAATTTCA CACAGGA). Quantitation of reverse transcription products was carried out with a PhosphorImager (Molecular Dynamics). All experiments were carried out a minimum of three independent times to ensure reproducibility of the data.

\section{Transient transfection and reporter gene assays}

Drosophila Schneider S2 adherent cells were cultured in Shields \& Sang M3 media (Sigma) prepared with yeast extract (Sigma) and bactopeptone (Difco) that was supplemented with 10\% heat-inactivated FBS. Cells were transfected in 24-well plates by using the Transfectol reagent (Gene Choice). For dual luciferase assays, cells were plated at $0.6 \times 10^{6}$ cells per each well of a 24-well plate $1 \mathrm{~d}$ prior to transfection. Cells were transfected with the indicated amounts of a Caudal expression vector that was supplemented, where necessary, with pAC control expression vector to give a total of $2.5 \mu \mathrm{g}$ of DNA of expression vector. In addition, the firefly luciferase reporter constructs (162 ng) were cotransfected with the Pol III-Renilla luciferase reporter (50 ng) (obtained from N. Perrimon, Harvard Medical School). Cells were harvested 20-26 h post-transfection and assayed for dual luciferase activities, as specified by the manufacturer (Promega). To correct for transfection efficiency, the firefly luciferase activity of each sample was normalized to the corresponding Renilla luciferase activity. Each transfection was performed in duplicate. The graphs represent an average of two to three independent experiments.

\section{Acknowledgments}

We thank W. McGinnis, T. Yusufzai, J. Theisen, B. Rattner, H. Ishii, D. Urwin, S. Torigoe, and S. Pitak for critical reading of the manuscript. We thank F. Furnari and W. Cavenee (Ludwig Institute for Cancer Research; University of California at San Diego) for the use of their luminometer; T. Yusufzai, D. Urwin, and W. McGinnis for helpful advice; and Norbert Perrimon (Harvard Medical School) for the generous gift of the Renilla luciferase control plasmid. This work was supported by a grant from the NIH to J.T.K. (GM041249).

\section{References}

Akam, M. 1987. The molecular basis for metameric pattern in the Drosophila embryo. Development 101: 1-22.

Brooke, N.M., Garcia-Fernàndez, J., and Holland, P.W. 1998. The ParaHox gene cluster is an evolutionary sister of the Hox gene cluster. Nature 392: 920-922.

Burke, T.W. and Kadonaga, J.T. 1996. Drosophila TFIID binds to a conserved downstream basal promoter element that is present in many TATA-box-deficient promoters. Genes \& Dev. 10: 711-724.

Burke, T.W. and Kadonaga, J.T. 1997. The downstream core promoter element, DPE, is conserved from Drosophila to humans and is recognized by TAFII60 of Drosophila. Genes \& Dev. 11: 3020-3031.

Butler, J.E.F. and Kadonaga, J.T. 2001. Enhancer-promoter specificity mediated by DPE or TATA core promoter motifs. Genes \& Dev. 15: 2515-2519.

Butler, J.E.F. and Kadonaga, J.T. 2002. The RNA polymerase II core promoter: A key component in the regulation of gene expression. Genes \& Dev. 16: 2583-2592.
Charité, J., de Graaff, W., Consten, D., Reijnen, M.J., Korving, J., and Deschamps, J. 1998. Transducing positional information to the Hox genes: Critical interaction of $c d x$ gene products with position-sensitive regulatory elements. Development 125: 4349-4358.

Davidson, A.J. and Zon, L.I. 2006. The caudal-related homeobox genes $c d \times 1 a$ and $c d \times 4$ act redundantly to regulate hox gene expression and the formation of putative hematopoietic stem cells during zebrafish embryogenesis. Dev. Biol. 292: $506-518$.

Davidson, A.J., Ernst, P., Wang, Y., Dekens, M.P., Kingsley, P.D., Palis, J., Korsmeyer, S.J., Daley, G.Q., and Zon, L.I. 2003. $c d \times 4$ mutants fail to specify blood progenitors and can be rescued by multiple hox genes. Nature 425: 300-306.

Dearolf, C.R., Topol, J., and Parker, C.S. 1989. The caudal gene product is a direct activator of fushi tarazu transcription during Drosophila embryogenesis. Nature 341: 340-343.

Deng, W. and Roberts, S.G. 2005. A core promoter element downstream of the TATA box that is recognized by TFIIB. Genes \& Dev. 19: 2418-2423.

Deng, W. and Roberts, S.G. 2007. TFIIB and the regulation of transcription by RNA polymerase II. Chromosoma 116: $417-$ 429.

Evans, R., Fairley, J.A., and Roberts, S.G. 2001. Activator-mediated disruption of sequence-specific DNA contacts by the general transcription factor TFIIB. Genes \& Dev. 15: 29452949.

FitzGerald, P.C., Sturgill, D., Shyakhtenko, A., Oliver, B., and Vinson, C. 2006. Comparative genomics of Drosophila and human core promoters. Genome Biol. 7: R53. doi: 10.1186/ gb-2006-7-7-r53.

Gehring, W.J. 1987. Homeo boxes in the study of development. Science 236: 1245-1252.

Gershenzon, N.I., Trifonov, E.N., and Ioshikhes, I.P. 2006. The features of Drosophila core promoters revealed by statistical analysis. BMC Genomics 7: 161. doi: 10.1186/1471-2164-7161.

Grenier, J.K., Garber, T.L., Warren, R., Whitington, P.M., and Carroll, S. 1997. Evolution of the entire arthropod Hox gene set predated the origin and radiation of the onychophoran/ arthropod clade. Curr. Biol. 7: 547-553.

Hoey, T., Doyle, H.J., Harding, K., Wedeen, C., and Levine, M. 1986. Homeo box gene expression in anterior and posterior regions of the Drosophila embryo. Proc. Natl. Acad. Sci. 83: 4809-4813.

Hsu, J.Y., Juven-Gershon, T., Marr II, M.T., Wright, K.J., Tjian, R., and Kadonaga, J.T. 2008. TBP, Mot1, and NC2 establish a regulatory circuit that controls DPE-dependent versus TATA-dependent transcription. Genes \& Dev. 22: 2353-2358.

Juven-Gershon, T., Hsu, J.Y., Theisen, J.W.M., and Kadonaga, J.T. 2008. The RNA polymerase II core promoter - the gateway to transcription. Curr. Opin. Cell Biol. 20: 1-7.

Kutach, A.K. and Kadonaga, J.T. 2000. The downstream promoter element DPE appears to be as widely used as the TATA box in Drosophila core promoters. Mol. Cell. Biol. 20: 4754-4764.

Lagrange, T., Kapanidis, A.N., Tang, H., Reinberg, D., and Ebright, R.H. 1998. New core promoter element in RNA polymerase II-dependent transcription: Sequence-specific DNA binding by transcription factor IIB. Genes \& Dev. 12: 34-44.

Lappin, T.R., Grier, D.G., Thompson, A., and Halliday, H.L. 2006. HOX genes: Seductive science, mysterious mechanisms. Ulster Med. J. 75: 23-31.

Lemons, D. and McGinnis, W. 2006. Genomic evolution of Hox 
gene clusters. Science 313: 1918-1922.

Levine, M. and Tjian, R. 2003. Transcription regulation and animal diversity. Nature 424: 147-151.

Levine, M., Harding, K., Wedeen, C., Doyle, H., Hoey, T., and Radomska, H. 1985. Expression of the homeo box gene family in Drosophila. Cold Spring Harb. Symp. Quant. Biol. 50: 209-222.

Lim, C.Y., Santoso, B., Boulay, T., Dong, E., Ohler, U., and Kadonaga, J.T. 2004. The MTE, a new core promoter element for transcription by RNA polymerase II. Genes \& Dev. 18: 1606-1617.

Lohnes, D. 2003. The Cdx1 homeodomain protein: An integrator of posterior signaling in the mouse. Bioessays 25: 971980.

Macdonald, P.M. and Struhl, G. 1986. A molecular gradient in early Drosophila embryos and its role in specifying the body pattern. Nature 324: 537-545.

Mlodzik, M. and Gehring, W.J. 1987. Expression of the caudal gene in the germ line of Drosophila: Formation of an RNA and protein gradient during early embryogenesis. Cell 48: 465-478.

Mlodzik, M., Fjose, A., and Gehring, W.J. 1985. Isolation of caudal, a Drosophila homeo box-containing gene with maternal expression, whose transcripts form a concentration gradient at the pre-blastoderm stage. EMBO J. 4: 2961-2969.

Nüsslein-Volhard, C. and Wieschaus, E. 1980. Mutations affecting segment number and polarity in Drosophila. Nature 287: 795-801.

Ochoa-Espinosa, A. and Small, S. 2006. Developmental mechanisms and cis-regulatory codes. Curr. Opin. Genet. Dev. 16: $165-170$.

Ohler, U., Liao, G.C., Niemann, H., and Rubin, G.M. 2002. Computational analysis of core promoters in the Drosophila genome. Genome Biol. 3: RESEARCH0087. doi: 10.1186/gb2002-3-12-research0087.

Ohtsuki, S., Levine, M., and Cai, H.N. 1998. Different core promoters possess distinct regulatory activities in the Drosophila embryo. Genes \& Dev. 12: 547-556.

Pearson, J.C., Lemons, D., and McGinnis, W. 2005. Modulating Hox gene functions during animal body patterning. Nat. Rev. Genet. 6: 893-904.

Rivera-Pomar, R. and Jäckle, H. 1996. From gradients to stripes in Drosophila embryogenesis: Filling in the gaps. Trends Genet. 12: 478-483.

Rivera-Pomar, R., Lu, X., Perrimon, N., Taubert, H., and Jäckle, H. 1995. Activation of posterior gap gene expression in the Drosophila blastoderm. Nature 376: 253-256.

Schulz, C. and Tautz, D. 1995. Zygotic caudal regulation by hunchback and its role in abdominal segment formation of the Drosophila embryo. Development 121: 1023-1028.

Smale, S.T. 2001. Core promoters: Active contributors to combinatorial gene regulation. Genes \& Dev. 15: 2503-2508.

Smale, S.T. and Kadonaga, J.T. 2003. The RNA polymerase II core promoter. Annu. Rev. Biochem. 72: 449-479.

Soeller, W.C., Poole, S.J., and Kornberg, T. 1988. In vitro transcription of the Drosophila engrailed gene. Genes \& Dev. 2: 68-81.

Wampler, S.L., Tyree, C.M., and Kadonaga, J.T. 1990. Fractionation of the general RNA polymerase II transcription factors from Drosophila embryos. J. Biol. Chem. 265: 21223-21231.

Wu, L.H. and Lengyel, J.A. 1998. Role of caudal in hindgut specification and gastrulation suggests homology between Drosophila amnioproctodeal invagination and vertebrate blastopore. Development 125: 2433-2442. 


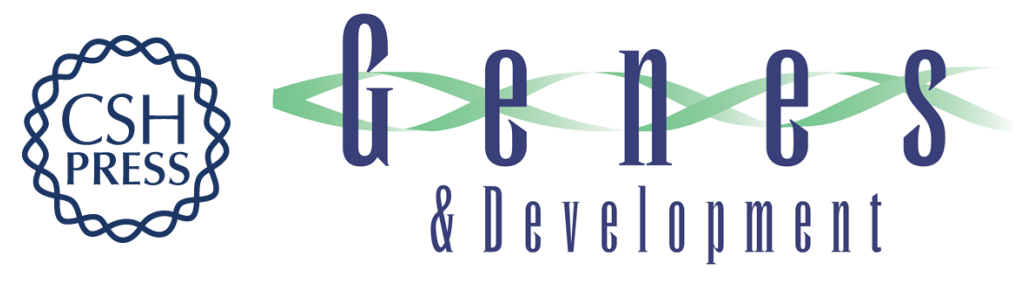

\section{Caudal, a key developmental regulator, is a DPE-specific transcriptional factor}

Tamar Juven-Gershon, Jer-Yuan Hsu and James T. Kadonaga

Genes Dev. 2008, 22:

Access the most recent version at doi:10.1101/gad.1698108

Supplemental http://genesdev.cshlp.org/content/suppl/2008/10/17/22.20.2823.DC1
Material

References This article cites 44 articles, 22 of which can be accessed free at:

http://genesdev.cshlp.org/content/22/20/2823.full.html\#ref-list-1

License

Email Alerting Receive free email alerts when new articles cite this article - sign up in the box at the top

Service right corner of the article or click here.

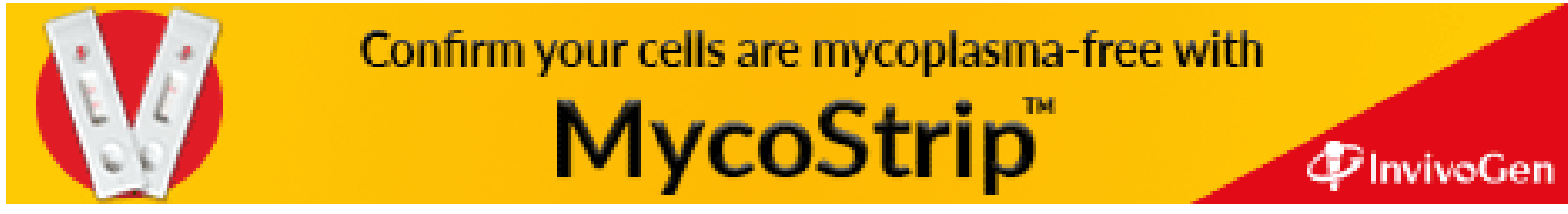

